\title{
ANALYSIS OF FREE ROAD DATA IN TANZANIA, UGANDA AND KENYA USING FREE AND OPEN SOURCE SOFTWARE
}

\author{
Stefan Jovanovic ${ }^{1}$, Dina Jovanovic ${ }^{1}$, Gorica Bratic ${ }^{1}$, Maria Antonia Brovelli 1, * \\ ${ }^{1}$ Politecnico di Milano, Department of Civil and Environmantal Engineering, Piazza Leonardo da Vinci, 20133 Milano, Italy - \\ (stefan.jovanovic, gorica.bratic, maria.brovelli)@polimi.it, dina.jovanovic@mail.polimi.it
}

Commission IV, WG IV/4

KEY WORDS: Free and open data, OpenStreetMap, roads, positional accuracy, completeness, road accessibility rate

\begin{abstract}
:
Roads are one of the most important infrastructural objects for each country. Slow development of third world countries is partially influenced by missing roads. Therefore, United Nation (UN) enlisted them inside the ninth Sustainable Development Goal (SDG) whose achievement highly relies on geospatial data. Since the authoritative data for the majority of developing countries are incomplete and unavailable, the focus of this study is on free data. The conveyed research, explained in this paper, was divided in two parts. The first one refers to completeness and positional accuracy assessment of three different road data sets (freely available). The second part was focused only on OpenStreetMap (OSM) since it showed the best results in the previous stage. Thus, OSM was used to compute (in the second part of the research) and analyse the road accessibility rate within the buffer zone of two kilometers from human settlements. To locate human settlements, raster data, representing land covers were used. Results are pointing where the infrastructure is not mapped or is not present. The complete work was done using Free and Open Source Software, which is important, since the proposed procedure can be implemented by anyone.
\end{abstract}

\section{INTRODUCTION}

Good infrastructure has a vast effect on the whole sustainable development of a country, ie. economy, industry and trade. The efficient road network, is a part of infrastructure that is required to maximize economic and social benefits of any country (Ivanova and Masarova, 2013).

The importance of having strong and well-structured network of roads is also shown through the United Nations' Sustainable Development Goals (UN SDGs) (https://doi.org/10.18356/29c75b3e-en) in which the ninth goal focuses on Industry, Innovation and Infrastructure. Each SDG is characterized by indicators that are frameworks for future actions and analysis. Particularly for goal nine, the indicator 9.1.1 considers the proportion of the rural population who live within $2 \mathrm{~km}$ of all-season roads. This indicator will address governments, stakeholders, and decision makers where they should invest in road infrastructure. In order to achieve its successful computation, as well as the similar ones, geospatial data related to population and roads are required. Since collection of those data is demanding, regarding both, money and time, free and open data were used in this research. However, before using those roads data for any kind of purpose their quality has to be examined and secured.

That fact raises the core research questions: "How to analyse free and open road data and how to accomplish the calculation of road accessibility rate (RAR)?" More specifically, the study aimed to achieve the following specific research objectives:

- To identify the most reliable free road data source;
- To create a workflow for computation of RAR (similar to SDG indicator 9.1.1) by exploiting just Free and Open Source Software (FOSS);

- To present results applying multi-dimensional visualization that is understandable for policymakers.

The area of interest is on three neighbouring African countries: Kenya, Tanzania and Uganda (Figure 1). They were chosen because the data model representing roads is the same for all of them and because interest in assembling and organizing free geospatial data is increasing in developing countries (Goodchild, 2007). Another fact was manifested by a growing number of volunteered geographic information (VGI) projects, collecting data in that region.

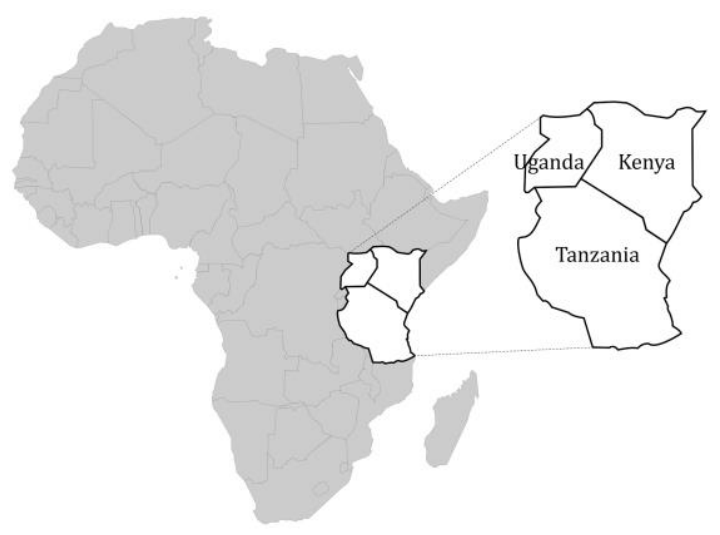

Figure 1. Area of interest in developing countries: Kenya, Uganda and Tanzania

* Corresponding author 
A good example of VGI project is Crowed2Map Tanzania (https://crowd2map.wordpress.com). This idea is based on the crowdsourced mapping, where volunteers are giving their contribution to Female Genital Mutilation (FGM) prevention by collecting roads data in rural areas of Tanzania. This leads to completion and creation of the maps that can, for instance, guide endangered girls to safe houses.

The research paper is structured as it follows: The first section introduces the area of interest, methodology and main research goals. Section 2 reviews related works with similar topics. Section 3 shows the positional accuracy and completeness analysis of three roads data sets. The most accurate one was used in Section 4 to compute the RAR. Section 5 summarizes the completed work and suggests steps for future researches.

\section{RELATED WORKS}

The quality analyses of free road data were topic of many researches. They consist of comparing a free data set (like OpenStreetMap-OSM) to the other data set (usually authoritative data or satellite imagery) which is considered as more accurate (Haklay, 2010). While several studies had the main focus just on the completeness (Zielstra et al. 2011) other compared geometries, thematic attributes of road network data and their positional differences (Ludwig et al., 2011). Siebritz and Sithole (2014) completed a qualitative and quantitative comparison between national mapping agency data in South Africa and OSM. Growing number of free and open data sets triggered comparative analyses, that can give insight which one is more reliable and which one is developing faster in terms of quantity/quality (Mooney and Corcoran, 2014; Neis and Zipf, 2012).

Some researches were focused on the analysis of attributes assigned to road data (Leitner and Arsanjani, 2017). For instance, features referring to the seasonality of roads were useful while investigating access of population to these roads (Nkomo et al., 2016). According to Vincent (2018), missing that piece of information partially caused the failure of study, conveyed by The World Bank Group, that developed Rural Access Index (equal to SDGs indicator 9.1.1) in 2006.

Free and Open Source Software for Geospatial (FOSS4G) can be used to assess the quality of open datasets (Brovelli et al., 2015). These softwares are available to everyone, and they can also support personalized development of specific extensions (Martinez-Llario et al., 2009). Using FOSS, the source codes are available publicly, so they offer higher degree of flexibility to broader group of users and researchers. (Brovelli et al., 2016)

\section{ANALYSIS OF FREE GEOSPATIAL DATA}

The first step in the analysis of Free Geospatial road data in this research was the evaluation of the completeness and positional accuracy. Completeness accuracy serves to measure the gap in data collection. Deficiency of data can cause partial view of the overall picture. This considers discrepancies between the evaluated data set and a data set that is considered to have sufficient completeness (e.g. high-resolution satellite imagery). "Positional accuracy is a measurement of the variance of map features and the true position of the attribute (Antenucci et al., 1991)." Positional accuracy and completeness are included in both standards: European (CEN, 1995) and US (USGS, 1990), (Table 1).

Data, that showed the greatest level of positional and completeness accuracy, were used in the second part of research for computation of RAR.

\begin{tabular}{|c|c|}
\hline \multicolumn{2}{|c|}{$\begin{array}{l}\text { European Standard (European Committee for } \\
\text { Standardization/Technical Committees - CEN/TC287) }\end{array}$} \\
\hline \multicolumn{2}{|l|}{ Lineage } \\
\hline \multicolumn{2}{|l|}{ - Usage } \\
\hline - Quality parameters: & $\begin{array}{l}\text { Primary accuracy: } \\
\text { - Positional accuracy } \\
\text { - Thematic accuracy } \\
\text { - Logical consistency } \\
\text { - Completeness } \\
\text { - Temporal accuracy } \\
\sim \text { Secondary parameters: } \\
\text { - Textual fidelity }\end{array}$ \\
\hline \multicolumn{2}{|c|}{ US Standard (Spatial DAta Transfer Standard-SDTS) } \\
\hline \multicolumn{2}{|c|}{$\bullet \quad$ Lineage } \\
\hline \multicolumn{2}{|l|}{ - Positional accuracy } \\
\hline \multicolumn{2}{|l|}{ - Attribute accuracy } \\
\hline \multicolumn{2}{|l|}{ - Logical consistency } \\
\hline - Completeness & \\
\hline
\end{tabular}

Table 1. Set of European and US Standards for analysis of geospatial data

\subsection{Visual analysis approach for road completeness and positional accuracy}

Three different free road datasets (Figure 2) were examined in this research :

- OpenStreetMap Roads (OSM);

- Road dataset published by Digital Chart of the World (DWC) and provided by United Nations Economic Commission for Africa (UNECA);

- Global Roads published by Columbia University's and provided by Centre for International Earth Science Information Network (CIESIN).

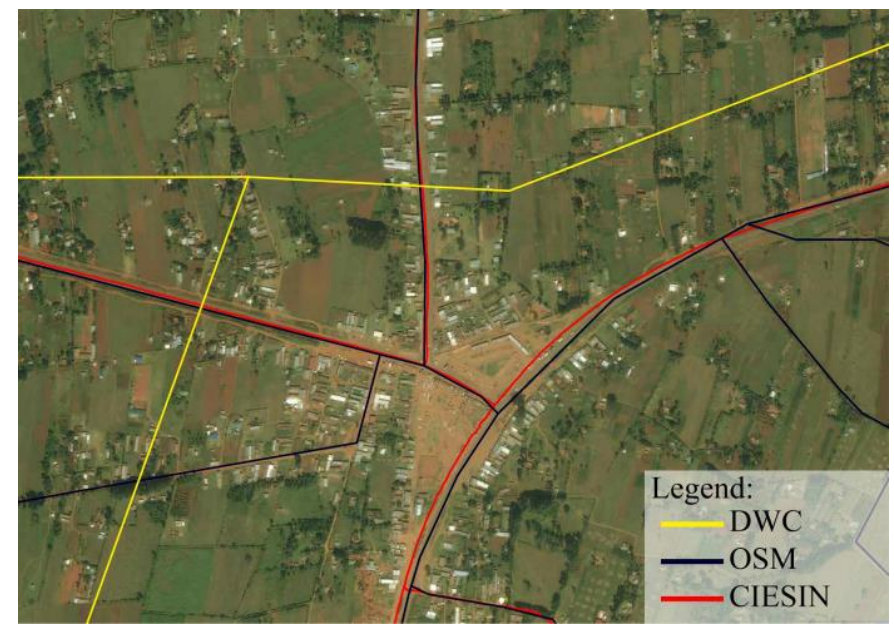

Figure 2. Randomly selected hexagon with three sets of road data

For each country in the area of interest ratios between total lengths of OSM and two other data sets (respectively) were 
computed. Values presented in Table 2 are giving the first insight how the completeness differs between these datasets.

\begin{tabular}{|c|c|c|}
\hline COUNTRY & TLosm/TLCIESIN & TLosm/TLDCw \\
\hline Kenya & 2.5 & 4.5 \\
\hline Tanzania & 5.6 & 7.7 \\
\hline Uganda & 6.5 & 8.0 \\
\hline
\end{tabular}

Table 2. Ratio between OSM and DCW road data and OSM and CIESIN road data

Yet, to have more reliable information about the completeness, visual inspection was done. The data to be analysed here are rather complex, thus they were divided into regular cells i.e. grid to simplify the procedure. There are various grid shapes, but only three have regular geometric form (equilateral and equal internal angles) that ensure continuity in a dataset. They are: equilateral triangle, square and hexagon. Among these shapes, the more similar to a circle the polygon is, the closer to the centroid the points near the border are (Moreira de Sousa and Leitão, 2017). Therefore, the area of interest was divided in hexagon grid with the apothem of $10 \mathrm{~km}$ that can give enough data inside boundaries but yet not too large. From total number of hexagons in all three countries is 4422, a sample of 220 randomly chosen hexagons was used for road data accuracy estimation, and the following procedure was repeated for each of them. A hexagon was used to represent a "bounding box" where three evaluators were checking road data accuracy, independently of each other. The assessment of the completeness of features was done by the visual comparison of road data sets with Bing Aerial imagery (hereafter satellite imagery) in QGIS.

Before performing visual "inspection", comparisons between temporal resolutions of satellite imagery and each road data set were accomplished.

Under assumption that complete data set does not exist, evaluators had the opportunity to assign one of the three attributes representing completeness:

- $\quad$ sufficient - existing road data were also visible on satellite imagery;

- $\quad$ excessive - road data existed, but could not be recognized on satellite imagery;

- no data - road data were missing.

The value assigned by at least two evaluators was considered as the final one for completeness of the data.

Regarding the estimation of positional accuracy, only hexagons where roads were labeled as sufficient were taken into account. At first, each evaluator was searching for the feature (usually crossroad) represented by the road data set which was recognizable on the satellite imagery. After that, the distance between same feature in data set and satellite imagery was assigned as the value for the positional accuracy. Since evaluators worked individually they chose different features to measure that distance. Finally, the positional accuracy of complete road data set was the mean of positional accuracies of individual hexagons.

\subsection{Results of visual analysis approach for road completeness and positional accuracy}

For all datasets majority of hexagons were classified as 'sufficient'. However, results in Table 3 are proving that OSM is the most complete, with 219 hexagons as 'sufficient' and just one hexagon classified as "excessive". CIESIN and DCW datasets contain $24 \%$ and $28 \%$ (respectively) hexagons that are missing data or assigned as "excessive".

\begin{tabular}{|c|c|c|c|}
\hline \multirow{2}{*}{ Completeness } & OSM & CIESIN & DCW \\
\cline { 2 - 4 } & \multicolumn{3}{|c|}{ Number of hexagons } \\
\hline Sufficient & $219(99.5 \%)$ & $173(76 \%)$ & $157(71 \%)$ \\
\hline Excessive & $1(0.5 \%)$ & $17(8 \%)$ & $36(16 \%)$ \\
\hline No data & 0 & $30(16 \%)$ & $27(12 \%)$ \\
\hline
\end{tabular}

Table 3. Number of hexagons per completeness classes

For each data set the mean value, median and standard deviation of positional accuracy were computed. Results presented in Table 4 are pointing out OSM as the positionaly most accurate dataset.

\begin{tabular}{|c|c|c|c|}
\hline Indicator & OSM & CIESIN & DCW \\
\hline Number of hexagons & 219 & 173 & 157 \\
\hline $\begin{array}{c}\text { Mean Value of positional } \\
\text { accuracy [m] }\end{array}$ & 35 & 600 & 1100 \\
\hline $\begin{array}{c}\text { Median of positional } \\
\text { accuracy [m] }\end{array}$ & 20 & 220 & 600 \\
\hline $\begin{array}{c}\text { Standard deviation of } \\
\text { positional accuracy [m] }\end{array}$ & 45 & 740 & 860 \\
\hline
\end{tabular}

Table 4. The basic statistics of positional accuracy

The analysis of statistics showed that OSM road data are the most reliable for further research. OSM road data are often enriched by information about road surface. Road surface categories can be generic, simply distinguishing between natural and man-made materials (unpaved and paved) on the road surface. On the other hand, more detailed categorization sets apart specific materials (e.g. asphalt or gravel), but eventually they can be assigned to one of the generic categories. In example of Kenya's roads, $56 \%$ of the roads are not labeled with the surface categories. The share of unpaved roads is $24 \%$ if only accounting for the roads explicitly labeled as unpaved. However, if dirt, gravel, ground and sand roads are added (as they are natural materials), the unpaved road share raises to 
$34 \%$. The remaining $10 \%$ of the roads in Kenya are paved. (Figure 3).

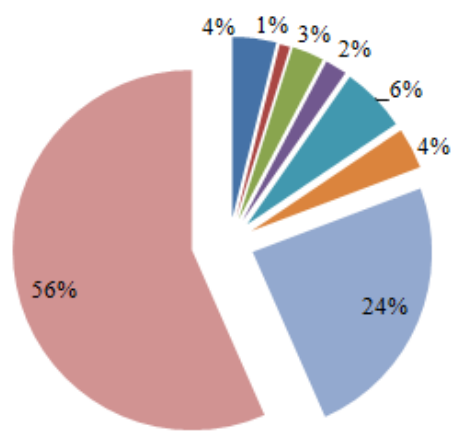

$$
\begin{aligned}
& \text { = asphalt } \\
& \text { = dirt } \\
& \text { m gravel } \\
& \text { m ground } \\
& \text { = paved } \\
& \text { = sand } \\
& \text { = unpaved } \\
& \text { = no data }
\end{aligned}
$$

Figure 3. Pie chart of surfaces for Kenya's secondary roads

\section{ROAD ACCESSIBILITY COMPUTATION USING LAND COVER RASTERS AND OSM ROAD DATA}

Three different rasters were used to extract information about areas in which there is human footprint (hereafter human settlement):

- $\quad$ Climate Change Initiative - S2 Prototype Land Cover 20m map of Africa 2016 (CCI+);

- GlobeLand30 (GL30);

- Global Human Settlement Built-Up Grid (GHS).

\subsection{Description of the used raster data}

CCI+(http://2016africalandcover20m.esrin.esa.int), developed by European Space Agency (ESA), represents 20 meters resolution land cover map of Africa based on 1 year of Sentinel2A observations from December 2015 to December 2016. Among 10 generic classes that appropriately describe the land surface the class Built-up areas is devoted to human settlement. The Coordinate Reference System used for the CCI+ is a geographic coordinate system based on the World Geodetic System 84 (WGS84) reference ellipsoid.

GL30 (http://www.globallandcover.com) is a product of "Global Land Cover Mapping at Finer Resolution" project led by the National Geomatics Center of China (NGCC). GL30 refers to land cover of the earth between latitude $80^{\circ} \mathrm{N}$ to $80^{\circ} \mathrm{S}$, containing 10 classes with different land cover types. Pixels representing human settlement (PHS) belong to class Artificial surface and their value is 80 . The resolution of GL30 is 30 meters and it adopts WGS84 coordinate system. For this paper, GL30 from reference year of 2015 was used.

GHS (https://ghsl.jrc.ec.europa.eu/ghs_bu.php), as a part of Global Human Settlement Layer project, is supported by the European Commission (EC), Joint Research Center and Directorate-General for Regional and Urban Policy. GHS is a 38 meter resolution binary raster in which values are expressed in byte from 1 (for non-built areas) to 101 (for built-up areas), with 0 representing no data. Data used for this paper refer to 2015 and they are in Spherical Mercator projection.
Table 5 summarizes the provider, the resolution, European Petroleum Survey Group (EPSG) code, the year of satellite imagery used to produce land cover map and PHS.

\begin{tabular}{|c|c|c|c|c|c|}
\hline Name & Provider & $\begin{array}{c}\text { Resolution } \\
{[\mathrm{m}]}\end{array}$ & $\begin{array}{c}\text { EPSG } \\
\text { code }\end{array}$ & Year & $\begin{array}{c}\text { PHS } \\
\text { value }\end{array}$ \\
\hline CCI+ & ESA & $20 \times 20$ & 4326 & 2016 & 8 \\
\hline GL30 & NGCC & $30 \times 30$ & 4326 & 2015 & 80 \\
\hline GHS & EC & $38 \times 38$ & 3857 & 2015 & 101 \\
\hline
\end{tabular}

Table 5. Characteristics of different raster data

The computation of population density was based on Worldpop (http://www.worldpop.org.uk) raster, which is a result of mapping project of numerous universities, agencies and organizations. This raster, whose resolution is 100 meters, EPSG 4326, represents distribution of human population for 2015.

\subsection{Procedure applied for human settlement extraction and RAR computation}

For each hexagon the RAR was computed (Equation 1), representing the ratio of length of roads inside $2 \mathrm{~km}$ buffer around the settlement and the area of settlement. Priorly, the algorithm (Figure 4) was developed and used to generate input parameters for its calculation.

$$
R A R=\text { L2km buffer } / A_{\text {settlement }}
$$

The development of algorithm and execution was done in Python (version 3.6.) programming language.

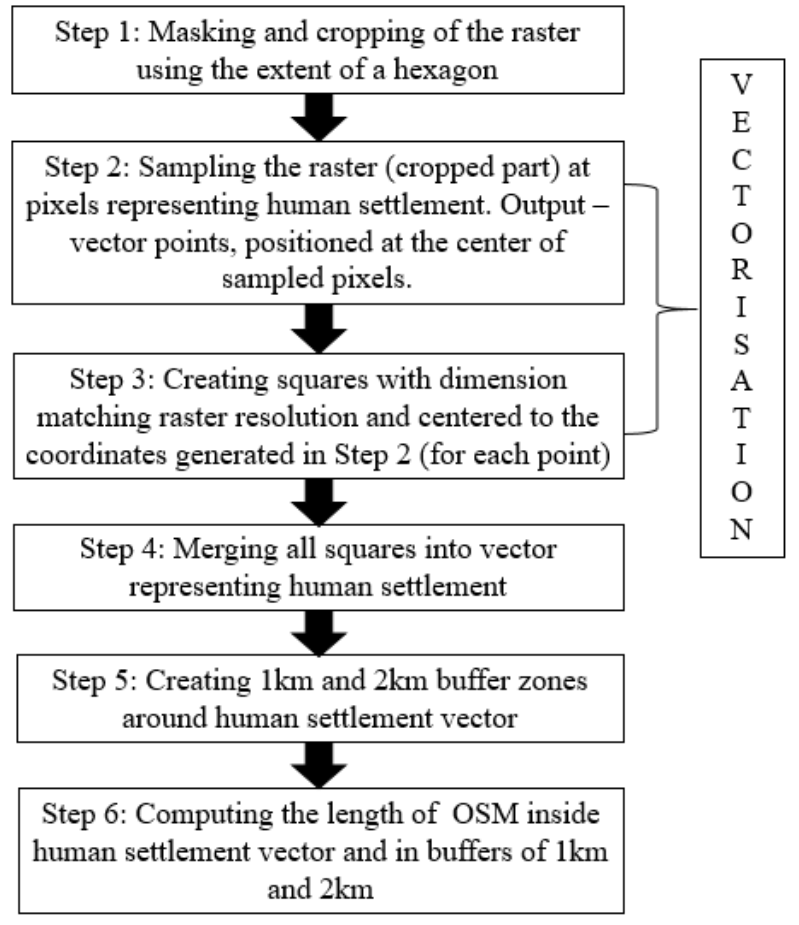

Figure 4. The algorithms workflow 
Step 1 is masking and cropping of the raster, by the extent of a hexagon. Steps 2 and 3 represent process of vectorisation. At first, the cropped part of raster is sampled at the points representing human settlement. As a result, vector points positioned in centers of those pixels are generated. Than, squares of size equal to the pixel size and centered to the coordinates are created. By merging the squares (Step 4) the human settlement is defined and afterwards buffer zones are built (Step 5). At the end the length of OSM inside settlement and two buffer zones is computed.

The algorithm includes following Python packages/libraries:

1. Rasterio (GDAL and NumPy Python library for geospatial raster data access)

2. Shapely (Package for manipulation and analysis of planar geometric objects)

3. Numpy (Package for scientific computations)

4. Geopandas (Package that enables user to do in Python that would otherwise require a spatial database such as PostGIS)

The computation of the surface of the human settlement for each hexagon (Step 4 - Figure 4), was included in the algorithm and executed simultaneously with its remainder.

\subsection{Results and multidimensional visualization}

For multidimensional interpretation of the results, the Qgis2threejs plugin of QGIS was used. For each hexagon the value $\mathrm{H}$ (Equation 2) was computed, representing the normalized value of RAR with respect to maximum value of RAR (for each country and land cover map individually).

$$
H=\left(R A R_{i} / R A R_{\max }\right) * 100
$$

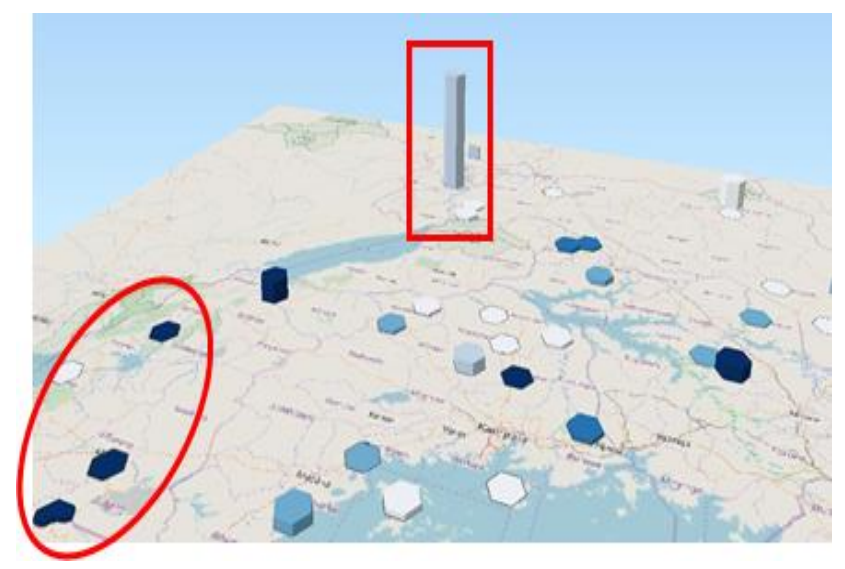

Figure 5. Human settlements "detected" by CCI+ for Uganda

Looking at Figure 5, one can notice different shades of blue. They are depicting different density of population per square kilometer. The darker the color is, the greater is the density. The height of hexagons is defined by normalized RAR value $(\mathrm{H})$. Its value goes from 0 to 100 . The hexagon in red rectangle (Figure 5 ) is showing the greatest value of $\mathrm{H}$. It means that the accessibility of roads within 2 kilometers around a human settlement 'detected' within CCI+ is good. It might happen that the detection of settlement using CCI+ is wrong, but in this particular case, computation of parameter $\mathrm{H}$ based on GL30 and GHS gave the same result $[\mathrm{H}=100]$ (check red rectangles on Figures 6 and 7).

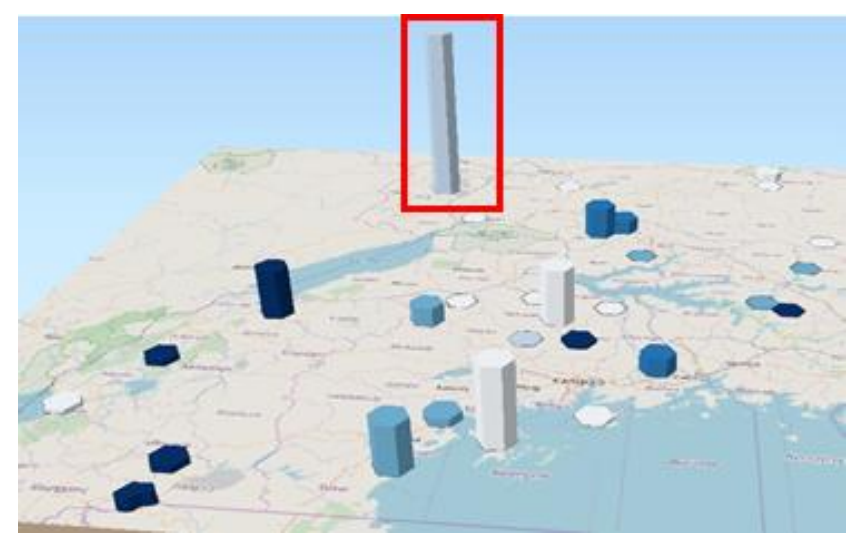

Figure 6. Human settlements "detected" by GHS for Uganda

Hexagons in ellipses (Figure 5) are showing small value of $\mathrm{H}$ for densely populated area. This is a critical situation. It means either lower RAR, or it could also mean that OSM road data are missing. The working methodology targets this particular situation for further investigation.

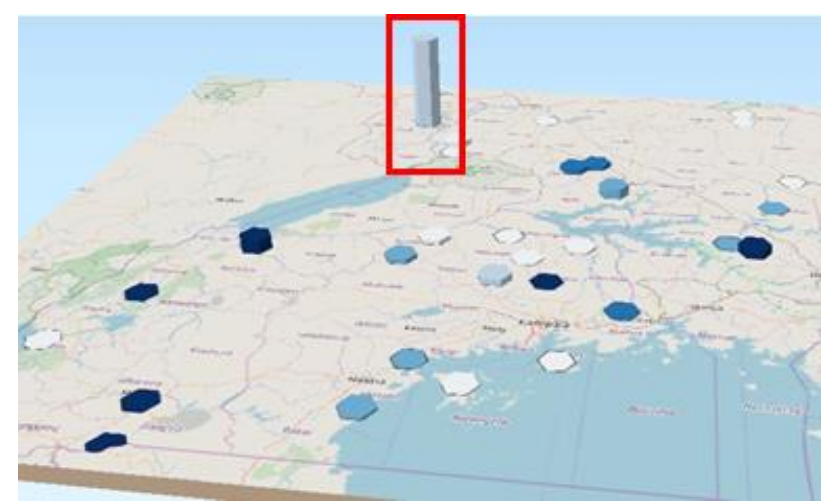

Figure 7. Human settlements "detected" by GL30 for Uganda

\section{CONCLUSION}

OSM, has proved to be the most complete and positinaly accurate out of 3 free geospatial data set evaluated in this research. Based on it, the RAR was computed and visualized in a such a way that one can understand where the road infrastructure has to be improved or where potentially OSM road data are missing.

Inclusion of additional data/parameters, like morphology of terrain, would give more realistic image of the RAR. For the purposes of this paper, just the roads in the horizontal plane were considered which means that, in our simplified model, no vertical obstacle exists between settlements and roads. Also, seasonal variability of roads has to be taken into account in future researches, since majority of roads are unpaved and their existence/usage highly depends on meteorological conditions.

Beside the assessment of positional/completeness accuracy and results of RAR, this paper promotes the usage of FOSS in GIS. The complete workload was carried out with QGIS and Python, using free geospatial data, which are available for everyone. This fact is important for the people in developing countries dealing with GIS, since they usually miss data and funds for investment in proprietary software. 


\section{ACKNOWLEDGEMENTS}

Special thanks to Andre Nonguierma and Girum Asrat (UNECA) for providing us data. Many thanks also to other data providers: OSM, ESA, NGCC, EC and CIESIN.

\section{REFERENCE}

Antenucci, J., Brown K., Croswell P., Kevany, M., Archer, H., 1991. Geographic Information Systems: A guide to the technology. Springer, New York, US. 102-103.

Brovelli, M.A., Minghini, M., Molinari, M., Mooney, P., 2015. A FOSS4G-based procedure to compare OpenStreetMap and authoritative road network datasets. Geomatics Workbooks 12, 235-238, ISSN 1591-092X.

Brovelli, M. A., Minghini, M. , Molinari, M. and Mooney, P., 2017, Towards an automated comparison of OpenStreetMap with authoritative road datasets. Transactions in GIS, 21: 191206. doi:10.1111/tgis. 12182

CEN, 1995, TC 287 N369: Geographic information - Data description - Quality. Technical report, European Committee for Standardization, Working Draft at stage 32, London, UK.

Goodchild, M. F., 2007. Citizens as sensors: the world of volunteered Geography. GeoJournal. 69, 211-221. doi.org/10.1007/s10708-007-9111-y.

Haklay, M., 2010. how good is volunteered geographical information? A comparative study of OpenStreetMap and ordnance survey datasets. Environment and Planning B: Planning and Design, 37(4), 682-703. https://doi.org/10.1068/b35097

Ivanova, E. and Masarova, J., 2013. Importance of road infrastructure in the economic development and competitiveness, Economics and Management, doi.org/10.5755/j01.em.18.2.4253.

Leitner, M. and Arsanjani, J.J., 2017. citizen empowered mapping, Springer Publishing Company, 188-200, http://dx.doi.org/10.1007/978-3-319-51629-5.

Ludwig, I., Voss, A., Krause-Traudes, M., 2011. Comparison of the street networks of Navteq and OSM in Germany, Advancing Geoinformation science of a changing world, Springer Berlin Heidelberg, 65-84, http://dx.doi.org/10.1007/978-3-642-197895_4.

Martinez-Llario, J., Coll, E., Arteaga, D., 2009. Road data analisys with FOSS GIS. Proceedings of the 9th WSEAS International Conference on Applied Computer Science, 191194, ISSN 1790-5109.

Mooney, P. and Corcoran, P., 2014. Analysis of interaction and co-editing patterns amongst OpenStreetMap contributors. $\begin{array}{llll}\text { Transactions in } & \text { 633-59, }\end{array}$ https://doi.org/10.1111/tgis.12051.

Moreira de Sousa, L. and Leitão, J. P., 2017. HexASCII: A file format for cartographical hexagonal rasters. Transactions in GIS. 10.1111/tgis.12304.
Neis, P. and Zipf, A., 2012. Analyzing the contributor activity of a volunteered geographic information project - The case of OpenStreetMap. ISPRS International Journal of GeoInformation. 1. 146-165. 10.3390/ijgi1020146.

Siebritz, L., Sithole, G., 2014. Assessing the accuracy of OpenStreetMap data in South Africa for the purpose of integrating it with authoritative data. PhD Thesis, University of Cape Town, SA.

https://pdfs.semanticscholar.org/2a9c/c10127fb5546269e77aed 6afe71df5235956.pdf.

Nkomo, S. L., Desai, S., \& Peerbhay, K. 2016. Assessing the conditions of rural road networks in South Africa using visual observations and field-based manual measurements: A case study of four rural communities in Kwa-Zulu Natal. Review of Social Sciences, 1(2), 42-55. doi:http://dx.doi.org/10.18533/rss.v1i2.24

Vincent, S., Civil Design Solutions, 2018. status review of the updated Rural Access Index (RAI), Draft Final Report, GEN2033C. London: ReCAP for DFID.

http://research4cap.org/Library/Vincent-CDS-2018StatusReviewUpdatedRAI-FinalReport_GEN2033C180529.pdf

Zielstra, Dennis \& Hochmair, Hartwig. 2011. Comparative study of pedestrian accessibility to transit stations using free and proprietary network data. Journal of the Transportation Research Board. 2217. 10.3141/2217-18. 